\title{
Elements of Geometric Theory of Complex Systems Behavior
}

\author{
YURY I. BRODSKY \\ Dorodnicyn Computing Centre \\ Federal Research Centre "Computer Science and Control” of RAS \\ 40, Vavilova street, Moscow, 119333 \\ RUSSIAN FEDERATION \\ yury_brodsky@mail.ru https://www.elibrary.ru/author_items.asp?authorid=99170
}

\begin{abstract}
The paper proposes the elements of a geometric theory of behavior. The basis of this theory is Model Synthesis - an end-to-end technology for describing, synthesizing and implementing agent models. Its main achievement - is the formalization of an agent by a mathematical object - a species of structure in the sense of N. Bourbaki. Algorithms that implement actions of agents are included in the base sets of this species of structure. Let us consider morphisms of the base sets with the special attention to the actions mappings. It occurs that unrestricted mappings of base sets can radically change system's behavior. We can use invariants as restrictions of mappings, if demand their preservation under admissible morphisms. As a result, we can obtain a classification of the systems behavior depending on the invariants that persist during the mappings.
\end{abstract}

Key-Words: - Complex Systems, Model Synthesis, Geometric Theory, Behavior, Species of Structure, Morphisms, Invariants, Conservation of Laws

Received: October 20, 2019. Revised: January 25, 2020. Accepted: February 10, 2020. Published: February 28, 2020.

\section{Introduction. On Complex Systems}

Let us outline the range of systems this work is devoted to, by listing some of their properties:

- Fractality. N.P. Buslenko in the work [1] calls "complex" a system, where it is easy to distinguish its components and these components themselves are complex systems.

- Behavior. A complex system and all of its components have behavior, - an ability to react in known methods on some conventional events of inner and outer environment.

- Openness. This means that a complex system exchanges streams of matter, energy, and information with the surrounding world.

- Nonequilibrium. Complex systems are dissipative in the sense of I. Prigogine [2].

- Three Worlds. To comprehend a complex system, we must consider it in three aspects: ideal, formal and material. We will discuss this issue in detail later.

- Dynamic Equilibrium and Sustainable Development. A complex system is sustainably developing while it is successful. However, its stability is dynamic. It should run for running as a bicycle. Moreover, as a rule a special control system is needed to keep this dynamic equilibrium. A section of this work is devoted to this point.

This work provides a language environment to describe and study complex systems behavior, as well as methods for its programming and modifying. We consider a class of simulation models represented by species of structure in the N. Bourbaki sense [3]. We consider the morphisms of the base sets of these structures and special attention is on the methods responsible for the model's actions. It occurs that unrestricted mappings of base sets can lead the system up to the opposite behavior. We can use invariants as restrictions of possible mappings, if 
demand their preservation from admissible morphisms. As a result, we can obtain a classification of the behavior of systems depending on the invariants that persist during the transformations of behavior.

\section{From Model Synthesis to the Geometric Theory}

Following N.P. Buslenko [1], we will distinguish components of complex systems. These components themselves can be complex systems in the sense of the previous sentence, i.e. consist of their own components, and so on. We stop this top-down analysis of the system at some level and assign the components of this level to be the agents of our model. We believe that everything is known about these agents: what they can do, their behavior, i.e. what they do in response to the possible events, how they are related and what information they exchange. The following problems arise

- To describe formally the existing knowledge about the complex system consisting of agents.

- To build an automatic synthesis of the complex system from the agents according to this description.

- To implement the synthesized simulation model of a complex system as a software package.

To solve this problem completely, the works [4, 5] proposed the concepts of Model Synthesis and Model-Oriented Programming. The central point of these concepts is a construction of universal agent for agent simulation - so called "model-component". A model-component is a formal mathematical object species of structure in N. Bourbaki sense [3]. It is a universal agent for agent simulation. The family of models-components has two important features

- The family of models-components is closed under the operation of uniting modelscomponents into the model-complex. The complex received by association of modelscomponents is a model-component itself, and therefore, can be included in new complexes.

- The organization of simulation calculations is same for all representatives of the family. This fact means a possibility of creation of the universal computer program capable to execute any simulation model, if that is the mathematical object supplied with the species of structure from that family of modelcomponents.

These properties of the models-components family permit to solve the problem of complex system synthesis from its agents. In addition, the concept of Model Synthesis gives some consequences of a very wide application.

First, we can match almost any agent complex system (physical or social) with its mathematical model (the model-component is a completely mathematical object of a certain species of structure), at least as a mental experiment. For the comparison, the author can suggest those who wish to try to match the political party or the social layer with its mathematical model using differential equations.

Secondly, N. Bourbaki's structures theory was initially oriented towards all kinds of mappings of base sets. This property of the structural theory was not necessary for solving the problem of synthesis of the agent model, but it turns out to be very important for studying the behavior of complex systems. First of all, we mean open dissipative systems in the sense of I. Prigogine [2]. Such systems exchange with the surrounding world flows of matter, energy, information. Mappings (morphisms) of the base sets of the complex systems structures are natural means of mathematical modeling of such exchanges.

The behavior of the model-component is composed of the possible actions it performs in response to the events - certain states of the internal and external environment. Recently, the author found out that some of scientists share his view on the behavior of a complex system, as an operating system built from separate basic actions [6].

Elementary actions and event determination functions belong to the base sets of the modelcomponent. Therefore, their morphisms can change the behavior of the system even to the opposite.

This fact opens up the possibility of constructing here a standard geometric theory [7]: limiting all possible mappings of base sets by certain invariants (axioms) and classifying the behavior of complex systems in accordance with the invariants preserved during admissible mappings.

So, this work is devoted to the construction of the elements of such a theory. 


\section{Elements of the Geometric Theory}

What does the author mean under the Geometric Theory? Structuralism has always been present in mathematics, but a conscious interest in it began with the Erlangen program [7], where F. Klein gave a classification of geometries known to him.

Take a projective plane, projective geometry defines a structure on it, projective transformations translate objects of projective geometry into equivalent ones (in the sense of this geometry). If we choose subgroups of the group of projective transformations that preserve one or another invariant, their elements transform objects into equivalent objects of the corresponding geometries. If we take the subgroup of congruent transformations (movements), we obtain the Euclidean geometry. If hyperbolic or elliptic metrics are invariant, we obtain a hyperbolic or elliptic non-Euclidean geometry. What the invariant is, - such is the geometry. We can classify geometries by invariants.

\subsection{N. Bourbaki’s Species of Structure}

Why at all species of structure? Now categories [8] or algebraic models [9] are much more popular among the scientists. The author, as a computer scientist, believes that a truly complex system always is to be considered and studied in three aspects - three worlds: the material world, the world of forms (projects, programs, control systems) and the world of ideas [10]. We know this research method since the times of Plato, but it undeservedly received the label of idealism in the Modern and Contemporary times.

\section{Species of structure consists of three parts}

- Base sets - the material world of species of structure.

- Typical characterizations - its world of forms.

- Axioms - the world of ideas.

Therefore, species of structure is a quite suitable instrument for modeling and studying of complex systems in the three worlds. Now let us move on to a simplified definition of the species of structure (without auxiliary base sets), sufficient for this work. A complete original definition can be found in [3].

N. Bourbaki's species of structure $\Sigma$, used in this work, has the following syntax: $\Sigma=<$ principal base sets; typical characterizations; axioms $>$ - three partitions are listed with a semicolon in the angle brackets.

The first - principal base sets $X_{1}, \ldots, X_{n}$, where these sets are listed with a comma.

The second - typical characterizations, where relations of the form

$$
\sigma_{1} \subset S_{1}\left(X_{1}, \ldots, X_{n}\right), \ldots, \sigma_{r} \subset S_{r}\left(X_{1}, \ldots, X_{n}\right)
$$

are listed with a comma. A set $\sigma$ is a species constant, and $S_{j}\left(X_{1}, \ldots, X_{n}\right)$ - so-called echelon construction. The echelon constructions are obtained by applying operations of Cartesian product $x$, and taking the set of all subsets $\beta(\cdot)$ to the original base sets and/or to the existing echelon constructions:

- By the definition $X_{i}$ is an echelon construction, for any $1 \leq i \leq n$.

- If $S$ is an echelon construction, then $\beta(S)$ (the set of all subsets of $S$ ) is an echelon construction.

- If $S$ and $S^{\prime}$ are echelon constructions, then $S \times S^{\prime}$ is an echelon construction.

- There are no other echelon constructions.

The third part of species of structure definition axioms

$$
R_{1}\left(X_{1}, \ldots, X_{n}, \sigma_{1}, \ldots, \sigma_{r}\right), \ldots, R_{l}\left(X_{1}, \ldots, X_{n}, \sigma_{1}, \ldots, \sigma_{r}\right)
$$

- arbitrary true statements over base sets and species constants. The only requirement for them is to preserve truth under isomorphisms of the base sets.

A few words about the original definition of the species of structure [3]. It is recursive - may require an already defined species of structure on auxiliary base sets (which are not subject of the future mappings). For example, to determine the species of structure of a linear vector space, we need an auxiliary species of structure of the algebraic field of real or complex numbers.

\subsection{Model-Component Species of Structure}

A family of models-components is defined as oneparameter family of species of structure, where parameter $N$ is a number of model's processes. The 
processes are formally determined by the typical characterizations (10) and axioms $R_{9}$ (indirectly, their definitions also include typical characterizations (8) with axioms $R_{8}$, determining the rules for elements switching in processes and typical characterizations (9), determining the initial elements of the processes.

$$
\begin{aligned}
& \Sigma_{N}=<X, M, E,\left\{M_{j}\right\}_{j=1}^{N},\left\{E_{j}\right\}_{j=1}^{N} \\
& x \subset X, a \subset X, \\
& s \subset M, f \subset M, \\
& \left\{m_{j, \text { real }} \subset M_{j} \times M\right\}_{j=1}^{N}, \\
& \left\{e_{j, \text { real }} \subset E_{j} \times E\right\}_{j=1}^{N}, \\
& \left\{m_{j, i n} \subset M_{j} \times \beta(X)\right\}_{j=1}^{N}, \\
& \left\{m_{j, \text { out }} \subset M_{j} \times \beta(X)\right\}_{j=1}^{N} \text {, } \\
& \left\{e_{j, i n} \subset E_{j} \times \beta(X)\right\}_{j=1}^{N}, \\
& \left\{s w_{j} \subset E_{j} \times M_{j} \times M_{j}\right\}_{j=1}^{N} \text {, } \\
& \left\{m_{j}^{0} \subset M_{j}\right\}_{j=1}^{N}, \\
& \left\{p_{j} \subset \beta\left(M_{j}\right) \times \beta\left(E_{j}\right) \times M_{j} \times \beta\left(E_{j} \times M_{j} \times M_{j}\right)\right\}_{j=1}^{N} ; \\
& R_{1}:(x \cup a=X) \&(x \cap a=\varnothing), \\
& R_{2}:(s \cup f=M) \&(s \cap f=\varnothing), \\
& R_{3}:\left\{\left(\forall m \in M_{j}\right)(\exists ! \tilde{m} \in M)\left(\{m, \tilde{m}\} \in m_{j, \text { real }}\right)\right\}_{j=1}^{N}, \\
& R_{4}:\left\{\left(\forall e \in E_{j}\right)(\exists ! \tilde{e} \in E)\left(\{e, \tilde{e}\} \in e_{j, r e a l}\right)\right\}_{j=1}^{N}, \\
& R_{5}:\left\{\left(\forall m \in M_{j}\right)(\exists ! r \in \beta(X))\left(\{m, r\} \in m_{j, i n}\right)\right\}_{j=1}^{N}, \\
& R_{6}:\left\{\left(\forall m \in M_{j}\right)(\exists ! r \in \beta(x))\left(\{m, r\} \in m_{j, \text { out }}\right)\right\}_{j=1}^{N}, \\
& R_{7}:\left\{\left(\forall e \in E_{j}\right)(\exists ! r \in \beta(X))\left(\{e, r\} \in e_{j, i n}\right)\right\}_{j=1}^{N},
\end{aligned}
$$

$$
\begin{gathered}
R_{8}:\left\{\left(\left(\forall e \in E_{j}\right)\left(\exists ! r \in M_{j} \times M_{j}\right)\left(\{e, r\} \in S w_{j}\right)\right) \&\right. \\
\left.\&\left(\left(\{e, r\} \in S w_{j},\{\tilde{e}, \tilde{r}\} \in S w_{j}, r=\tilde{r}\right) \Rightarrow(e=\tilde{e})\right)\right\}_{j=1}^{N}, \\
R_{9}:\left\{p_{j}=\left\{M_{j}, E_{j}, m_{j}^{0}, s w_{j}\right\}\right\}_{j=1}^{N},
\end{gathered}
$$

$R_{10}$ :- axiom of uniqueness of the modelcomponent characteristics calculating,

$R_{11}$ : - axiom of the behavior of the modelcomponent (organization of simulation calculations)>.

Everywhere $\left\{\cdots_{j}\right\}_{j=1}^{N}$ means that the contents of the braces is repeated with a comma $N$ times, while the index $j$ is replaced by numbers $1, \ldots, N$. For example, $\left\{M_{j}\right\}_{j=1}^{N}$ there is a shortened version of the record $M_{1}, \ldots, M_{N}$.

Principal base sets of the species of structure are $X, M, E,\left\{M_{j}\right\}_{j=1}^{N},\left\{E_{j}\right\}_{j=1}^{N}$. There are no auxiliary base sets. Further, $X-$ is the set of characteristics of the model, occasionally, we will divide it into two subsets $X=\{x, a\}$, where $x-$ are the internal characteristics of a model that, in accordance with the closeness hypothesis completely determine its status, and, $a$-its external characteristics, that by the same closeness hypothesis completely defines its interaction with all the rest world. $M$ - the set of different implementations of methods-elements and $M=\{s, f\}-$ slow $s$, realizing a smooth dependence of the internal characteristics of the model from its internal and external characteristics, and fast $f-$ which implement the internal model characteristics gaps. $E$ - the set of different implementations of methods-events, associated with the model. Events are what our model must respond to. The methodevent - is a method that gets as an input a subset of model characteristics $Y \subset X$, and gives as an output a non-negative number, indicating that the event occurred if the output is zero, or the forecast time-toevent, if the output is positive.

Each process $p_{j}, j=1, \ldots, N$, defined by (10) and $R_{9}$, successively carries out some finite set of elementary actions $M_{j}$, possible for it, which we will call the set of its methods-elements. The execution of one or another method-element depends on the 
situations arising in the system $E_{j}$, to which the process is able to respond, they will be called the set of its methods-events. Typical characterizations (3), (4) shows, from where the methods-elements and methods-events are taken, (5) - (7) shows what characteristics of the model are transmitted to methods-elements and accepted from them, typical characterization (8) sets the rules for switching elements under the influence of the events. Axioms $R_{1}-R_{8}$ complement and clarify the typical characterizations (1) - (8).

Note that the above view of the model-component is not an arbitrary invention of the author, but rather (as was shown in [5]) a necessary consequence of the closeness hypothesis, the main assumption that makes it possible to construct a simulation model.

What is the point of describing simulation models in the language of species of structures? Such a description makes it quite simple to prove in the language of the set theory that combining a finite number of models-components into a modelcomplex gives to it the same structure. Therefore, a model-complex is itself a model-component [4]. This is one of two principal features of the modelscomponents family.

The other principal feature is the same way of organizing simulation calculations for any member of the family. Again, the organization of calculations is not an arbitrary invention of the author, but a consequence of the fact that during finite simulation time we can find the model path only in the class of piecewise continuous functions (calculate it in a finite number of points). At each step of the simulation, we must:

- To check if at this step, there is a gap of the model trajectory, and if so, calculate it.

- To estimate the time of continuous evolution of the model. If the estimation is less than the simulation step, decrease the step.

- To calculate the continuous evolution of the trajectory at the simulation step.

- To transfer the model time and go to the next simulation step.

This way we act all the time of modeling, regardless of the type of model, its size, subject area, etc. More details on this can be found in $[4,5]$. Now we can tell in details, how the axiom of the behavior of the model-component $R_{11}$ works:
First, we select a standard simulation step $\Delta t$.

Secondly, it is believed that at the beginning of the modeling step, the current elements of all processes and all the internal characteristics of the model are known (in the first step, these are the initial values of the internal characteristics and the initial elements of the processes).

Thirdly, we assume that the external characteristics of the model can be determined at any moment at model time. Further

1. We compute the events associated with the current elements of all the processes. The rules of switching (8) determine correlation of events with the current elements of the processes. We can compute the events in parallel, but to promote the computing process further, we are to wait for completion of the computation of all the events. If there are events occurred, it is checked whether there are transitions to the fast elements from the sets $\left\{f_{j}\right\}_{j=1}^{N}$. If there are any - the fast elements run (they become current). We also can compute them in parallel, but to advance the computing process further, we are to wait for completion of all the calculations of the fast elements, and then return to the beginning of the item 1. If there are no transitions to fast elements - we transit to the new slow elements from $\left\{s_{j}\right\}_{j=1}^{N}$, and then return to the beginning of the item 1.

2. If there is no occurrence of the events, from all the forecasts the nearest $\Delta \tau$ is selected.

3. If the standard step of modeling $\Delta t$ does not exceed the predicted time to the nearest event $\Delta t \leq \Delta \tau$, - we compute the current slow elements with the standard step $\Delta t$. Otherwise, we compute them with the step to the nearest predicted event $\Delta \tau$. The model existence theorem proved in [5] gives us a chance not to fall into an infinite loop, decreasing the modeling step. Slow elements from the set $\left\{s_{j}\right\}_{j=1}^{N}$, can also be computed in parallel, with expectation of completion of the latter.

4. Return to the beginning of the item 1 .

Finally, we emphasize the two most important properties of the family of models-components: 
- We can organize the calculations of any member of the models-components family by the same universal computer program, which implements the $R_{11}$ axiom described above.

- Combining a finite number of family representatives into a complex according to certain rules [4], does not take the resulting construction beyond the family.

The indicated properties solve the problem of synthesizing a complex fractal multi-agent system from the individual agents.

\subsection{Mappings of the Behavior}

The most important property of the modelcomponent is that it has its own behavior, i.e. the ability to respond to certain pre-known influences (events) of the internal and external environment in one or another known way (method-element). This behavior is pre-programmed mainly by the typical characterizations (8) - (10) with axioms $R_{8}-R_{11}$, by choice of base sets $M$ and $E$, and partly by the typical characterizations (3), (4) with axioms $R_{3}, R_{4}$

. In fact, all the parts of the model-component description one way or another are involved in the programming of its behavior, but first of all, - the ones listed above.

We obtain the behavior of our complex system, having described the behavior of all modelscomponents and having constructed the synthesis of all the complexes, including the complex of the highest level. Now morphisms of base sets, especially $M$ and $E$ (since this is simpler and more understandable, but, possibly, also the others), can be interpreted as changes in the behavior of the system.

Changes (reprogramming) of the behavior of a complex system, their study, modeling and classification are very relevant. We often have to change our behavior. For example, getting a new job or getting a new task on the old one, creating new and changing old social connections, etc.

This work only raises this problem. The author does not have a ready mathematical solution, although there are some hints, analogies, and an understanding of the problem at the humanitarian level. We will offer examples from both mathematics and the field of social systems as an illustration of this understanding. The latter may seem to some people journalism, but the author would prefer to consider them a humanitarian analysis. Moreover, we can confirm this analysis by simulation models, where, thanks to the model synthesis, we may conduct the discourse in terms of the species of structures, morphisms, and invariants.

The first example is the equivalence of little men with clasped and disconnected hands, widely known from the algebraic topology. See the fig. 1 below.
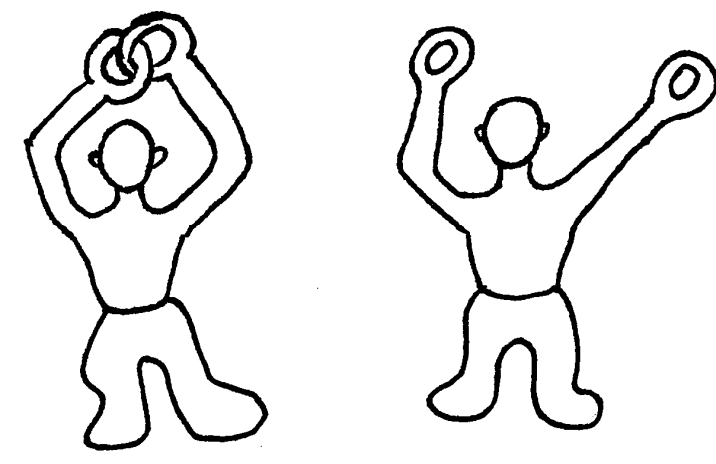

Fig.1. Topologically equivalent men

The fig. 2 below proves the topological equivalency of the little men from the above picture.
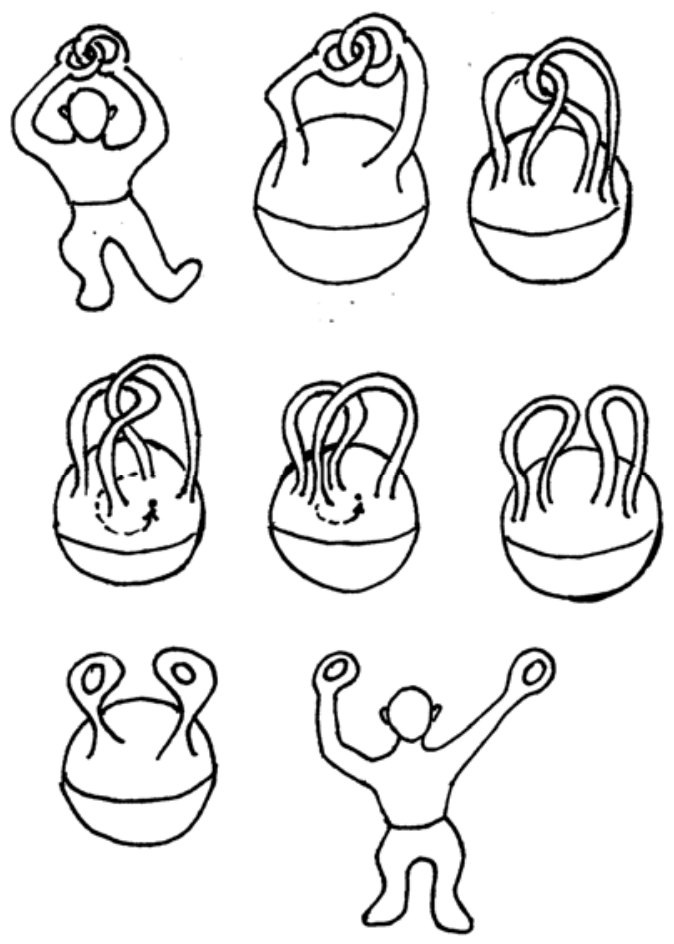

Fig. 2. The proof of the equivalency 
We consider little men in the category of topological spaces and treat as equivalent those that can be mapped into each other using a sequence of homeomorphisms, - continuous in both directions isomorphisms of the mentioned category.

The sixth picture - a ball with two "handles" - is a standard representative of the equivalence class generated by homeomorphisms, to which both little humans belong.

As the second example, let us cite the recent popular Overton windows [11]. (Joseph P. Overton, 1960 - 2003, American lawyer, political scientist, electrical engineer by the bachelor education. He himself called them the windows of discourse. They began to call them the windows of Overton after his tragic death in a plane crash). Overton windows offer technology for changing public behavior. They usually illustrate the work of this technology by the inclusion in public life of something hitherto completely unthinkable, such as cannibalism. The behavior mappings are discretized, divided into a finite number of windows, so that inside each window the actions and statements do not differ too much from each other in terms of proximity to existing norms and at the same time, gradually bring the discourse to the next window. They usually call the windows as follows:

1. Unthinkable.

2. Radical.

3. Acceptable.

4. Sensible.

5. Popular.

6. Policy.

Not all the sociologists consider Overton's window theory to be scientific. Some say they have not seen a single implementation in practice. The author can remind them that the great computer scientist Alan Turing was convicted for gross indecency in 1952, despite of his outstanding deserts to the British crown and world science. Just in 15 years, homosexuality in England was decriminalized, and now Britain is one of the most liberal countries with respect to LGBT people. A little over 50 years have passed.

Others say that the diversity of individuals and social strata does not fit into the concept of Overton's windows. This remark is also untenable, but its analysis is instructive. In the language environment of model synthesis there is an individual behavior of agents (models-components of the lowest level), there is collective behavior (a complex of the highest level), and there is the behavior of complexes of intermediate levels (for example, social layers). Morphisms are applicable, generally speaking, to any model-component, but usually they attribute Overton windows to the collective behavior of the entire system. Note that in this example, the natural language attempts to mix up individual, group, and system-wide behavior, but the language of geometric theory allows us to distinguish among them.

Let us comment the examples given.

The first example is a small fragment of algebraic topology, a branch of mathematics that developed rapidly in the twentieth century. Nevertheless, we did not see any mathematical formulas; all the proofs were reduced to the pictures. The author never have seen the homeomorphism formulas shown in this figure (although it is possible that he was poorly searching). However, it is obvious that we can write such formulas. They will be cumbersome (likely, heavier than the description of the model-component) and painful both for writing and for perception. Moreover, at the same time they will not add even a drop of understanding of the processes occurring in homeomorphisms in comparison with the pictures.

This example shows that in some cases, for a completely mathematical discourse, a natural language or even a language of pictures may be more adequate than the language of formulas. Mathematics from this, however, does not cease to be mathematics.

As for the Overton's windows, they are also mathematics, in our opinion, although to some they may seem journalism or even conspiracy theory. Apparently, in the space of actions (the basic set $M$ in the description of the model-component) one can introduce the topology of proximity in the public perception of certain actions. For example, at the domestic level, we often like to assess the proximity of actions (especially of other persons) to social norms. If topology is introduced, then Overton windows will be homeomorphisms (continuity of mappings is important) over the topological space of possible actions.

Morphisms can lead the system to the behavior opposite to the original, gradually, imperceptibly and in a not too long time. Therefore, the question naturally arises about invariants that limit the entire set of possible morphisms and about the classification of the behavior types associated with these invariants. 


\subsection{Invariants as Restrictions of Mappings}

The above example convinces us that unlimited morphisms can take the system very far from the original state. The traditional way to limit them is to choose invariants preserved by admissible morphisms. How do invariants work? The invariant, which is an axiom in the language of describing the structure of a complex system, distinguishes from the group of morphisms a subgroup of admissible morphisms preserving this invariant.

In the example of topologically equivalent men, such an invariant can be a wrist bracelet (in the fig. 3 below). No homeomorphism makes it possible to untangle the clasped hands if one of them has a bracelet. You can unfasten the rings of hands, but then the bracelet connects them.
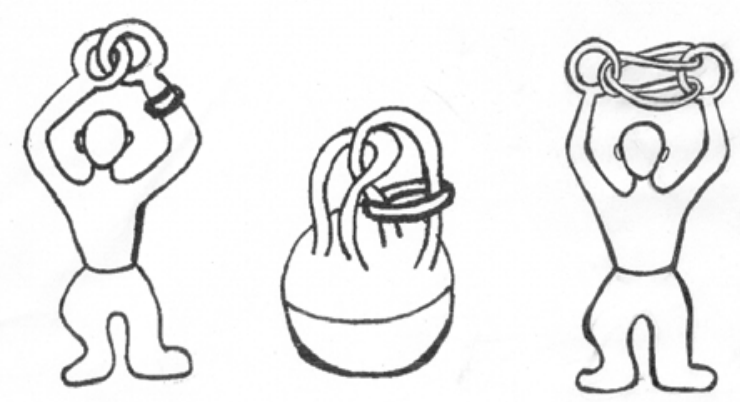

Fig. 3. A bracelet restricts hand disengagement

Invariants that restrict the transformation of behavior in society are traditionally religious commandments, moral and cultural values, traditional norms of behavior, which we call the Law with a capital letter. The Law is not always exclusively religious in its nature; it may include completely secular, as the ancient primordial traditions of a particular culture, as well as new constructivist norms.

As long as the Law is firmly rooted in public consciousness, Overton's windows cannot work successfully in a direction contrary to it - this will immediately cause public rejection and censure. For example, as long as society considers religious precepts to be a moral invariant, no Overton windows can make drug use, same-sex marriage, promiscuity, or even cannibalism a social norm.

Overton's windows did not fully work in this direction till the twentieth century, when religious invariants were recognized by the public consciousness as obsolete remnants of the "times of ignorance," restricting the freedom of personal expression, although the first unpunished attacks on them began from the Modern times.

From the above it follows that certain axioms invariant with respect to possible morphisms must be satisfied so that the behavior of a complex system under the influence of these morphisms changes only in the desired directions.

\subsection{Dynamic Equilibrium and Conservation of Laws in Complex Systems}

Unlike the closed physical systems dominated by conservation laws, the complex systems studied here are usually open and dissipative in the sense of I. Prigogine [2]. S.P. Kurdyumov's school studied systems of that kind in our country. They paid the main attention to the non-linearity of laws describing these systems, and possibility of blow-up regimes for them. Such systems exchange their base sets with the environment; therefore, morphisms of base sets are a quite adequate means of studying these systems. Complex systems are far from thermodynamic equilibrium, but they are in dynamic equilibrium: in order to work, they must work (such as a four-stroke engine, or any enterprise). To maintain dynamic equilibrium, a complex system must firstly maintain the potential of dynamic equilibrium - to perform a certain minimum work per unit of time (for example, at least 800 revolutions per minute for the internal combustion engine, paying salaries, utilities, renting premises, etc., for the enterprise). The author's teacher, Yu.N. Pavlovsky in many of his works (for example, [12]) noted that complex systems tend to maintain their structure.

Now let us see what the theory of agent modeling can give to study the stability of more complex models. For example, consider a university. Morphisms of the basic sets of an educational institution are changes of generations of students, teachers also change, but usually for longer times. In addition, the cast of the employees and administration of any enterprise, and even the building and the place where this enterprise is located may vary trough the time. Nevertheless, the enterprise remains "the same" in the perception of all those who deal with it.

What makes it the same? - This is its structure, expressed in regulatory documents (for example, the charter, the list of duties, labor contracts), as well as 
unwritten corporate laws and traditions. Moreover, both written and unwritten programs of behavior are based on the invariants - axioms on which the structure of an organization is built.

Why is the structure preserved? In order to function, a dissipative system needs to spend part of its power on maintaining the process of its functioning, as well as on maintaining its structure, including the functionalities that are not currently involved. The simplest example is a four-stroke internal combustion engine. All the work is performed on the combustion cycle and should be sufficient for at least the remaining cycles - exhaust, collection and compression of the mixture, as well as the operation of the fuel pump, cooling system, lubrication, etc. Equality of work performed and fixed costs occurs at the engine idle speed. Therefore, it is impossible to force the engine to work at lower speeds - it just stops. For the engine to spin, it must spin! This is dynamic equilibrium. To start the engine, you need a special external effect of the starter - a device that has no relations with the already running engine. The starter gives to the engine the potential of dynamic equilibrium, only having which it can work. As medieval alchemists used to say: "In order to make gold, you must have gold".

Something like this, only a little more complicated, the situation is with any complex dissipative system. Just like a motor, such a system must work in order to work. Its equilibrium is also dynamic. The potential of dynamic equilibrium will be the sum of costs per unit of time to maintain the structure of a complex system. For example, if we are talking about an enterprise, we need to pay salaries to employees, including units that are not directly related to the organization functionality, such as bookkeeping, security, business services, etc. It is necessary to pay loans, rent premises and equipment, communication services, heating, electricity, and maintain production facilities in a "combat" state.

Above we mention the costs of maintaining the material constituent of the structure of a complex system. In addition to them, there may be parts related to the worlds of information and ideas. An enterprise is to maintain a corporate culture of relations and production (first of all, it is just the preservation of the invariants of the organization under morphisms of personnel behavior, the most common of which is the replacement of personnel), preservation and expanding the customer network, staff training, communication with educational institutions supplying personnel, and much more. Although this part of the structure is not material, the costs of its conservation are quite material and enter the potential of dynamic equilibrium.

For an enterprise begin to work, you need to give it the potential of dynamic equilibrium, for which there are special mechanisms that not relate directly with the functioning of the enterprise, for example, attracting investors, bank credit, or shares issuing.

If for a sufficiently long time, the income of the enterprise falls below the potential of dynamic equilibrium, it will become bankrupt, will stop and cease to exist in its former quality. Most likely, that its base sets (all kinds of assets) will go to other enterprises for next to nothing.

Let us now dwell on the methods of maintaining the behavior of a complex system. In a simpler case of a computer-controlled technical system, it will be a system of scheduled work to check the hardware and software. In social systems, such routine work also takes place, but they differ somewhat in form and name. We will call a cult the system of periodic events aimed at maintaining invariants and behaviors. Separate events of the cult - rituals, separate actions of rituals - ceremonies. The cult should program social behavior to preserve invariants and focus on the positive results of their conservation. According to the famous Russian mathematician, priest, philosopher and poet P.A. Florensky (for example, [13]), cult is the basis of the culture.

Perhaps someone will object that the cult is something completely religious. The author will not argue with this, indeed, the projection of religion on the ethological level of the culture has similar tasks, but he notes that there may not be any religion here. To preserve the invariants of behavior, it is enough to periodically program "adherence to certain symbolic systems," as the famous anthropologist Mary Douglas put it in [14].

For example, in a completely secular USSR, there was an extensive system of Communist party, Komsomol, Pioneer and even October organizations of all levels, with regular meetings of primary organizations, meetings of committees and bureaus of all levels, as well as various other ceremonies and rituals - elements of a cult. The main task of this cult was to maintain the invariants and structure of Soviet society. As we see, this cult did not cope with its task.

Perhaps, not least because this cult was not reflected as a cult in the public consciousness, and first of all in the consciousness of its servants. They rarely considered their role as servants of the cult and 
all the time rolled down to more understandable for them, materialists, production and business tasks, where they often only interfered with work and discredited the existing system.

Elements of the cult are easy to notice in the practice of modern large corporations, force structures, as well as educational and scientific organizations.

As a result, we can say that the potential of dynamic equilibrium is the price that a complex system pays per unit of time to remain itself, to preserve and maintain its structure. For this price, it gets the constancy of the laws by which it lives.

Therefore, we can say that in a complex system, instead of conservation laws, conservation of laws takes place. The system tries to preserve the conditions of its existence. However, physical conservation laws are a special case of the conservation of laws described above. They follow from symmetries - the consequences of the conservation of certain invariants (energy - the uniformity of time, momentum - the uniformity of space, etc.).

\section{Conclusion}

Based on the methods of model synthesis, it is possible to approach the geometrical theory of behavior. This theory provides a mathematical language environment for discourse in the subject field of modeling social behavior, capable of identifying subtle differences of the considered entities that are sometimes lost when they discuss in natural language.

In a complex system, the conservation of laws ensures its sustainable existence and development maintaining its dynamic equilibrium in the ideological, informational and physical planes. Violation of the dynamic equilibrium ceases the existence of a complex system in its former quality.

Using the language of geometric theory, it is shown that in order to maintain the given behavior of a complex system, it is not enough to take physical actions to maintain the potential of dynamic equilibrium (except for the well-known simple cases). An ideological-informational system for programming the conservation of invariants providing a given behavior is definitely needed. In this work it is called a cult.
The cult, along with maintaining the potential of dynamic equilibrium, ensures the conservation of the laws of a complex system existence. Known physical Conservation Laws are a special case of the Conservation of Laws. The phenomenon of Conservation of Laws in a complex system confirms the opinion of P.A. Florensky [13], that the cult is the basis of the culture.

In the work [15], there was an attempt to classify the types of ethno-cultural behavior according to several primordial invariants preserved in some different cultures.

Perhaps the style of this work may seem not rigor, close to journalism. However, it should be borne in mind that behind each proposed "humanitarian" construction its mathematical model may well stand, constructed by the method of model synthesis and, therefore, represented by a completely formal mathematical object - the model-component. This makes it possible to conduct formal mathematical discussions in terms of the species of structure, morphisms of base sets, invariants preserved by these morphisms, etc.

\section{References:}

[1] N. P. Buslenko, Complex systems and simulation models, Cybernetics, Vol. 12, No 6, 1976, pp. 862-870.

[2] I. Prigogine, G. Nicolis, Self-Organization in Non-Equilibrium Systems, Wiley, 1977.

[3] N. Bourbaki, Elements of Mathematics. Theory of Set, Springer, 2004.

[4] Yu. I. Brodsky, Bourbaki's Structure Theory in the Problem of Complex Systems Simulation Models Synthesis and Model-Oriented Programming, Computational Mathematics and Mathematical Physics, Vol. 55, No 1, 2015, pp. 148-159.

[5] Yu. I. Brodsky, Model synthesis and modeloriented programming - the technology of design and implementation of simulation models of complex multicomponent systems, In the World of Scientific Discoveries, Series B, Vol. 2, No 1, 2014, pp. 12-31.

[6] A. Cohn, M. A. Maréchal, D. Tannenbaum, C. L. Zünd, Civic honesty around the globe, 
Science, Vol. 365, No 6448, 2019, pp. 70-73. DOI:10.1126/science.aau8712.

[7] F. Klein, A comparative review of recent researches in geometry, https://arxiv.org/abs/0807.3161, last accessed 2019/12/11.

[8] S. Mac Lane, Categories for the Working Mathematician, Springer, 1998.

[9] S. Foldes, Fundamental Structures of Algebra and Discrete Mathematics, John Wiley \& Sons, 1994.

[10] Yu. I. Brodsky, On Mathematical Modeling in the Humanities, Power, Violence and Justice: Reflections, Responses and Responsibilities. View from Russia: collected papers XIX ISA World Congress of Sociology (Toronto, Canada, July 15-21, 2018), Moscow: RSS; FCTAS RAS, 2018, pp. 46-64.

[11] A brief explanation of the Overton window, Mackinac Center for Public Policy, https://www.mackinac.org/OvertonWindow, last accessed 2019/12/11.

[12] Yu. N. Pavlovsky, Fundamentals of mathematical modeling for complex systems, In: System Analysis and Modeling of Integrated World Systems. Vol. 1, EOLSS Publishers Co. Ltd, 2009, pp. 221-234.

[13] P. A. Florensky, The Pillar and Ground of the Truth: An Essay in Orthodox Theodicy in Twelve Letters, Princeton (NJ) University Press, 2004.

[14] M. Douglas, How Institutions Think, Syracuse (NY) University Press, 1986.

[15] Yu. I. Brodsky, Russia - the West or the East? Mathematical Models and Humanitarian Analysis, The Futures We Want: Global Sociology and the Struggles for a Better World. View from Russia: collected papers. The $3^{\text {rd }}$ ISA Forum of Sociology "The Futures We Want: Global Sociology and the Struggles for a Better World”, Moscow: RSS, 2016, pp. 53-59. 